\title{
EXPERIENCES IN GROWTH RETARDATION WITH HEAVY VITALLIUM STAPLES
}

\author{
Kenneth C. McGibbon, Alfred E. Deacon, Winnipeg, Canada, \\ and Clifford C. Raisbeck, San Francisco, California, United States of America \\ From the Shriners Hospital for Crippled Children and the Children's Hospital, Winnipeg, Canada
}

Stapling of the epiphysial plates at the knee to compensate for growth disturbances is frequently used and frequently maligned. It is often unjustly feared. The premises in this paper are that stapling is easy to perform, that it is as easy to perform badly, and that, when done with proper care in suitable children, it gives pleasing results. We have carried out stapling operations in seventy children-on a total of 136 epiphysial plates-between February 1949 and April 1960. This paper is based on that experience. For the past three years large Vitallium staples have been used. The operations were performed by a number of surgeons. We have had no experience with correction of angulation deformities with staples.

\section{INDICATIONS}

The prime indication for growth retardation by stapling is the presence in a growing child of a length discrepancy which causes deformity or disability. By giving the patient two short limbs, stapling must not make the patient more grotesque than he would be in an elevated shoe. If the discrepancy when the child is first seen is equal to or greater than the possible correction by growth arrest, a permanent epiphysiodesis is recommended. Such an operation at least decreases the lift on the shoe or the amount of femur to be resected at maturity-an important consideration. Growth arrest of any kind is associated with less morbidity and a shorter stay in hospital than formal bone shortening by resection. Moreover, epiphysial stapling usually incurs less post-operative care than does permanent arrest, although a second admission to hospital is necessary to remove the staples. The advantages of stapling in relation to growth prediction are discussed in another section of this paper.

Both the tibia and the femur are stapled unless the opposite femur is alone responsible for shortening. Then the femur only is done. Tibial stapling alone does not often yield sufficient correction, although, as pointed out by Ratliff (1959), most children with leg discrepancy after poliomyelitis have more tibial shortening than femoral; total length correction is more important than knee level.

\section{ESTIMATING CORRECTION}

Blount (1958) stated that actuarial tables were not reliable in predicting the growth of a diseased limb or in predicting the correction to be expected by growth arrest. In our experience consistently less correction was obtained by stapling than was to be expected from reference to the various prediction charts. This was true whether stainless steel or Vitallium staples were used in groups of three or two on each side of the plate. It is possible that stapling causes an early acceleration of growth until compression occurs. Other authors agree that initial arrest is slow, but our deficiency in correction amounted to more than the 0.6 centimetre mentioned by Green and Anderson (1957). Therefore, because growth can be resumed when needed, stapling should be done when wrist radiographs indicate a skeletal age of nine or ten instead of later. That is, temporary arrest must be done well before the actuarily estimated time - as soon as the bones are sufficiently mature to hold a staple well. This leaves a margin which has proved necessary because all correction depends upon growth of the diseased limb. 
Revision of the graph estimate with figures obtained from the individual is also difficult owing to the inconsistency of any coefficient over a period of time (Green 1949). As maturity approached in our patients we have worried much more that there would te insufficient correction than that growth would resume after removal of staples. So rarely were staples removed while significant growth potential remained that not enough data are available for statistical analysis of growth resumption. This is unfortunate, but the evidence of others seems valid (Blount and Clarke 1949, Blount and Zeier 1952, Dalton and Carpenter 1954, Blount 1958). The observation of frequent under-correction is not new, for Phemister made it in 1945; few have emphasised it. Green and Anderson's (1957) series of carefully measured and checked children showed that out of forty-four stapled at the actuarily correct time there were none with over-correction and five with under-correction. Five out of 125 children treated by epiphysiodesis needed arrest of the short side to halt over-correction, while eight fell short of the predicted mark.

Estimation of final correction is based on the well documented thesis that there is a slight overgrowth when staples are removed but that this amount is cancelled out by fusion of the epiphysis three to six months early due to insults to its temperament (Blount and Zeier 1952). It is probably easier to estimate what a well leg will do after the removal of staples than what a diseased one will do at any time.

Of course all reconstructive surgery must be completed as early as possible and certainly before the time for removal of staples. Surgery can shorten an extremity by a centimetre or two or perhaps lengthen it by stimulation.

If a deformity is non-progressive, such as overgrowth from a fracture years before, the staples are withdrawn when the extremities are just even. For other conditions an amount of over-correction necessary for counteracting progress is estimated by calculation of a growth decrement; or, if the patient is too old for prolonged observation, an intelligent guess is made, based on the natural history of the disease. Reckoning must also include the fact that growth decrements tend to revert towards normal as the original cause becomes more remote and as rehabilitation of the limb makes it a more usable member (White 1949). The intelligent guess is necessary for whatever type of growth arrest is undertaken, because the only way to obtain absolute accuracy is to await maturity and resect a measured portion of femur, accepting the associated increase in risk and morbidity. Finally, most orthopaedic surgeons feel that a permanent limb difference of one-half inch or less is not often clinically important (Straub, Thompson and Wilson 1945).

Measurements are made by radiographs as well as clinically by tape measure and elevation blocks. Radiographs are useful to establish the decrement and to observe the rate of correction, but certainly the final estimation for removal of staples must be made clinically by observance of stance and gait as well as by measurements. This is especially true if the cause of the shortening is not actual growth retardation but an asymmetric pelvis or hip dysplasia. Similarly, an arthrodesed knee or flail foot may influence the gait and require more or less than absolute length correction. Patients with lock knee braces or weak hip abductors benefit from one or two centimetres of shortening.

\section{THE OPERATION}

Our surgical technique is essentially that described by Blount and Clarke (1949) with the elimination of the "blind" insertion of staples, and substituting two Vitallium staples for the three stainless steel staples in each incision. The essential aid is a pair of steel spring calipers developed by Dr A. E. Deacon (Fig. 1). The spring tension should be just enough to allow the prongs to cling firmly to a child's femur or tibia. Such calipers, one somewhat larger than the other, can be easily made by any bracemaker. The Vitallium staples have broad flat points, reinforced shoulders, and are considerably heavier than the steel staples. The prongs are seven-eighths of an inch long and seven-eighths or five-eighths of an inch apart.

VOI. 44 B, NO. 1, FEBRUARY 1962 
We have never paid any attention to the fibular epiphysis but in no case was overgrowth of the fibula even noticeable, let alone a problem. Neither have we ever replaced that portion of the fibular head removed to permit the insertion of the posterior staple on the lateral side of the tibia.

Using a pneumatic tourniquet, four oblique incisions are made with the knee somewhat flexed. The two incisions on each side resemble a $\mathrm{V}$ but at least one inch of skin is left intact at the posteriorly placed apex. The centre of each incision should cross the epiphysial plate. Muscles and tendons are retracted, fascia is cut in the direction of its fibres and periosteum is exposed. The incisions shift somewhat with knee motion and skin retraction, thus affording access to the whole metaphysis. Capsule and synovium are avoided.

With a narrow osteotome the anterior third of the head of the fibula is resected and discarded, care being taken not to injure the common peroneal nerve or anterior tibial artery.

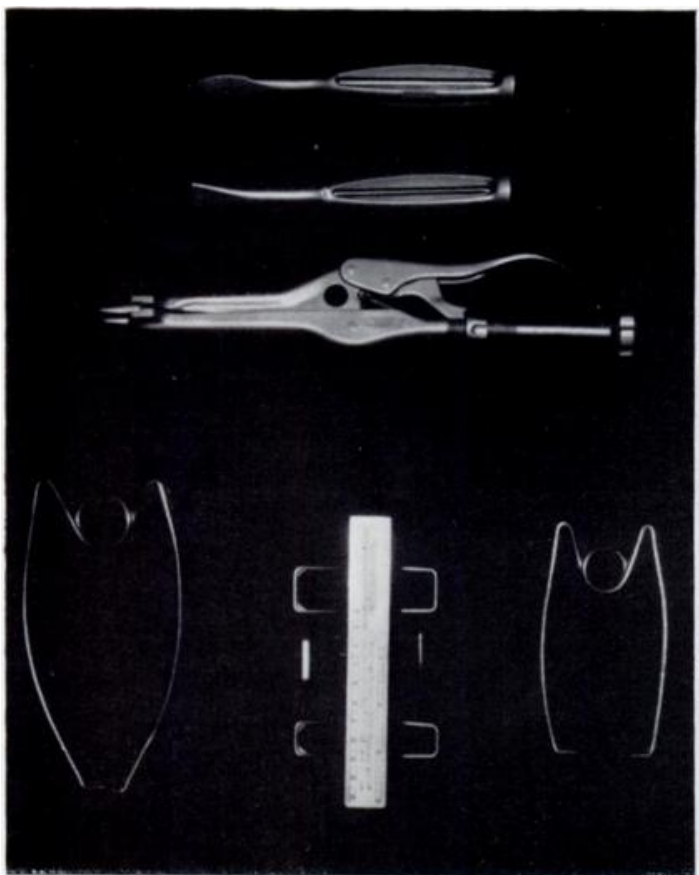

FIG. 1

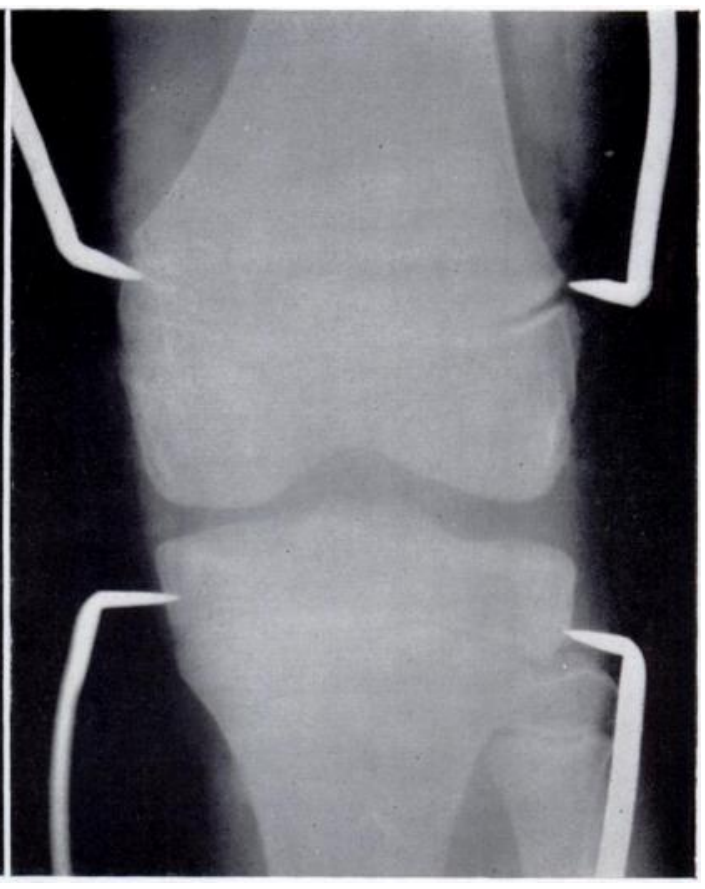

FIG. 2

Figure 1-The special instruments and staples. From above: staple remover, set, "vice-grip" holder and driver. At the bottom the larger femoral caliper is on the left and the smaller tibial caliper is on the right. Vitallium staples on the left are contrasted with the steel staples on the right. Figure 2-Antero-posterior radiograph showing calipers in place before the insertion of the staples.

The calipers are now placed with the prongs at the estimated position of the epiphysial plate. Perfect accuracy is not needed. The lateral tibial plate is usually even with the superior tip of the fibula. The femoral plate is just proximal to the apex of each condyle. A single antero-posterior radiograph is taken without projection errors, to show the exact relationship between the caliper prongs and the cartilage epiphysial plate (Fig. 2). The slope of the plates toward the joint can also be checked. With the caliper remaining engaged large Vitallium staples are placed astride the plate and parallel to its slope and are driven about half way in. We prefer the vice-grip type of holder and driver shown in Figure 1.

Two staples are used in each incision, the smaller five-eighths inch size in the tibia, and the seven-eighths inch size in the femur. The tibial staples must be placed well posteriorly to prevent genu recurvatum; the first lateral staple is inserted with the driver lying on the remains of the head of the fibula. The tips of all staples are pointed towards the centre of the marrow cavity to 
preclude projection into the popliteal space and to ensure good compression. Knee flexion makes this orientation easier. The staples are also aimed toward the joint to the degree determined by the slant of the epiphysial plate on the radiograph. Thus the prongs lie parallel to the epiphysial cartilage and a snug fit of the cross bar against the outflare of the tibial plateau or femoral condyle is ensured. Staples are placed symmetrically about one-half inch apart. More of the transverse bar of the staple should overlie the epiphysis than the metaphysis to avoid any possible damage to the germinal layer of cartilage cells. Antero-posterior and lateral radiographs are then taken with the same care as before (Fig. 3). In reading these films the direction of the staples towards the marrow cavity and towards the joint, and their position symmetrically on the metaphysis and sufficiently posterior in the tibia, must be perfect to be acceptable. The prongs must stop a little away from the joint as effusion and pain may

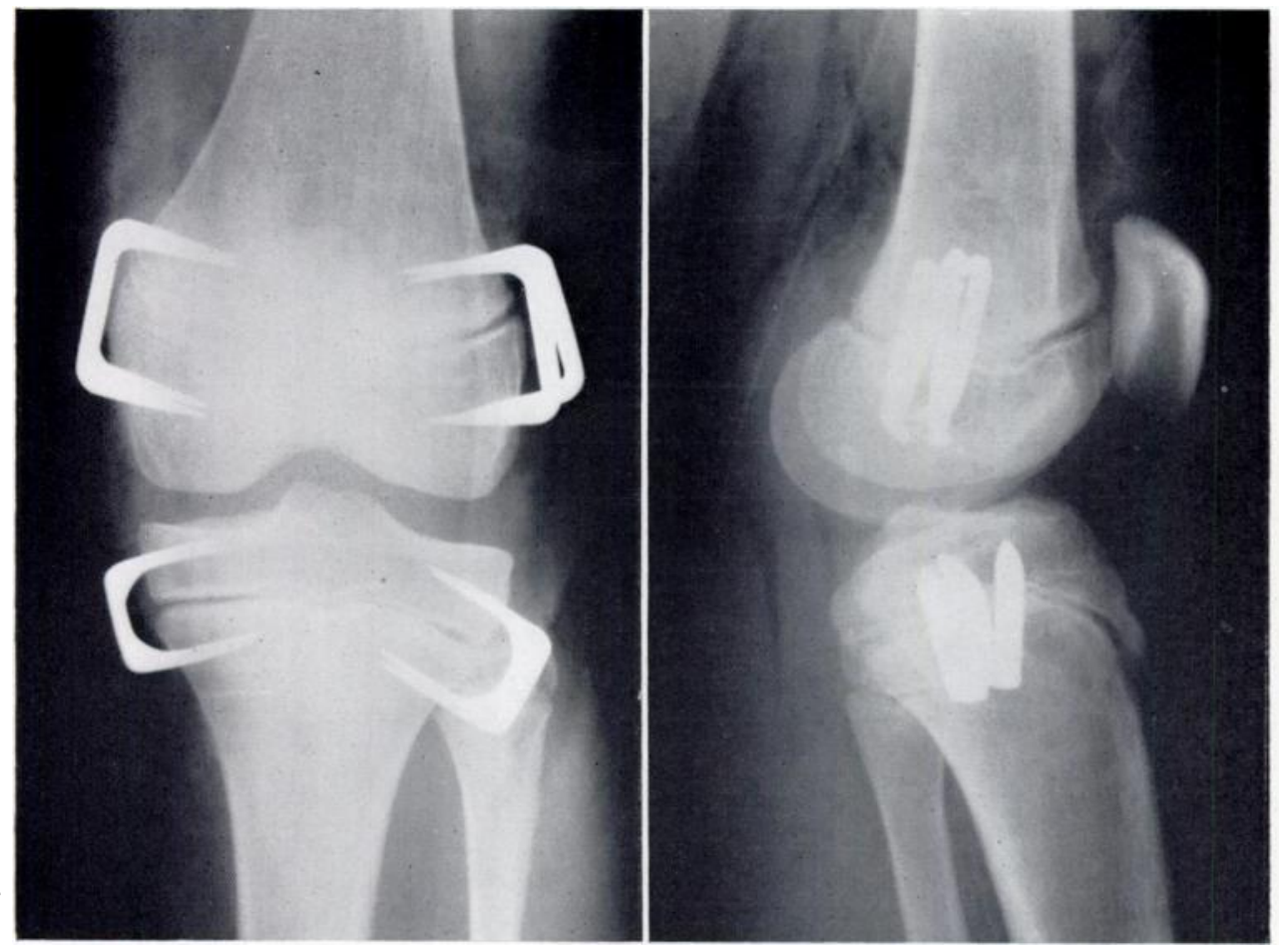

FIG. 3

Antero-posterior and lateral radiographs showing staples partly inserted in a satisfactory position and direction.

follow if they reach the articular cartilage. All being well, the staples are driven home with the set (Fig. 1) without further damage to the periosteum. A firm fit of staple to periosteum and bone is necessary to prevent extrusion. To ensure free knee motion no tissue except periosteum can be impaled. Because only two staples are used it is usually quite easy to stay extracapsular.

The wounds are then closed in layers with special care to obtain good subcutaneous apposition and eversion of skin edges to keep the scars narrow. A long leg cylinder cast is applied for three weeks to prevent spreading of the healing wounds by knee flexion during early ambulation. Children invariably regain full flexion when the cast is removed, usually faster without than with physiotherapy.

The use of the calipers reduces the number of radiographs, allows the surgeon to place the staples correctly the first time, and decreases the damage to periosteum and cartilage plate by the villain of this piece, the "searching surgeon."

VOl. 44 B, NO. 1, FEBRUARY 1962 
Only two staples are used in each incision because the strength of the heavier staples is sufficient to resist growth pressure without spreading. The flat prongs eliminate cutting through the plate and the tendency to extrusion is reduced since Vitallium is more firmly gripped by bone over a period of time than is steel.

It must be stressed that good results are directly correlated to the close attention paid to all the details mentioned.

\section{RESULTS}

Various errors in timing and technique led to disappointment in some of the earlier patients. As experience was gained the number of complications dropped and the accuracy of correction rose. A large number of the more recent patients, in whom Vitallium staples,were

TABLE I

Results of Stapling the Femoral and Tibial Epiphyses

\begin{tabular}{|c|c|c|c|c|c|c|c|}
\hline Total number of patients & . & - & . & . & . & 70 & \\
\hline \multicolumn{4}{|c|}{ Number of epiphysial plates stapled } & & . & 136 & \\
\hline \multicolumn{4}{|c|}{ Number of patients who have completed growth } & . & . & 44 & \\
\hline \multicolumn{3}{|c|}{ Patients lost, later amputated, etc. } & . & . & . & 10 & \\
\hline \multicolumn{6}{|c|}{ Adjusted number of patients who have completed growth } & 34 & \\
\hline \multicolumn{2}{|c|}{ Corrected to within one-half inch } & • & . & . & . & 26 & 76 per cent \\
\hline Insufficient correction. & . & - & . & . & . & 8 & 24 per cent \\
\hline Over-correction. & . & $\cdot$ & . & . & . & 0 & 0 per cent \\
\hline
\end{tabular}

TABLE II

Results by Type of Staple

\begin{tabular}{|c|c|c|c|c|c|}
\hline Completed cases using three steel staples & . & . & . & 20 & \\
\hline Corrected to within one-half inch & . & . & . & 15 & 75 per cent \\
\hline Insufficient correction . & . & . & . & 5 & 25 per cent \\
\hline Completed cases using two Vitallium staples & & . & . & 14 & \\
\hline Corrected to within one-half inch & . & & . & 11 & 79 per cent \\
\hline Insufficient correction & . & & . & 3 & 21 per cent \\
\hline
\end{tabular}

used, are correcting very well and will eventually swell the percentage of good end results. The number of complications on these patients is already much smaller. Each complication can be traced directly to some violation of the technique outlined above. Finally, it must be noted that although extrusion of a staple is distressing, if discovered early and replaced no serious or permanent harm has occurred. Therefore, aside from insufficient correction of length, there are no final bad results from complications in this series.

The greatest amount of correction obtained was one and a quarter inches. No patient suitable for stapling has presented young enough at our clinics with greater discrepancy; so this cannot be considered the greatest obtainable correction. Most of the patients reported here were operated upon at an older age than we now recommend, which perhaps accounts for some of the failures of complete correction (Tables I and II). 


\section{THE PROBLEM OF COMPLICATIONS}

It has been aptly said that bad results from stapling are the result of bad stapling. In the earlier patients stainless steel staples were used after the first published technique of Blount and Clarke (1949). Numerous miscarriages occurred until the technique of using spring calipers and heavier staples was introduced (Table III).

Possible complications include failure of arrest, genu valgum, genu varum and genu recurvatum caused by spreading, loosening, pulling through, corrosion, or fracture of the staples (Blount and Clarke 1949; Blount and Zeier 1952; Brockway, Craig and Cockrell 1954: Green and Anderson 1957; Goff 1960). Also listed but rarely seen is premature fusion of the plate with consequent over-correction. If large staples are inserted precisely as described these purely mechanical difficulties can be avoided. No permanent knee stiffness or serious infection occurred in our cases.

TABLE III

Complications of Epiphysial Stapling

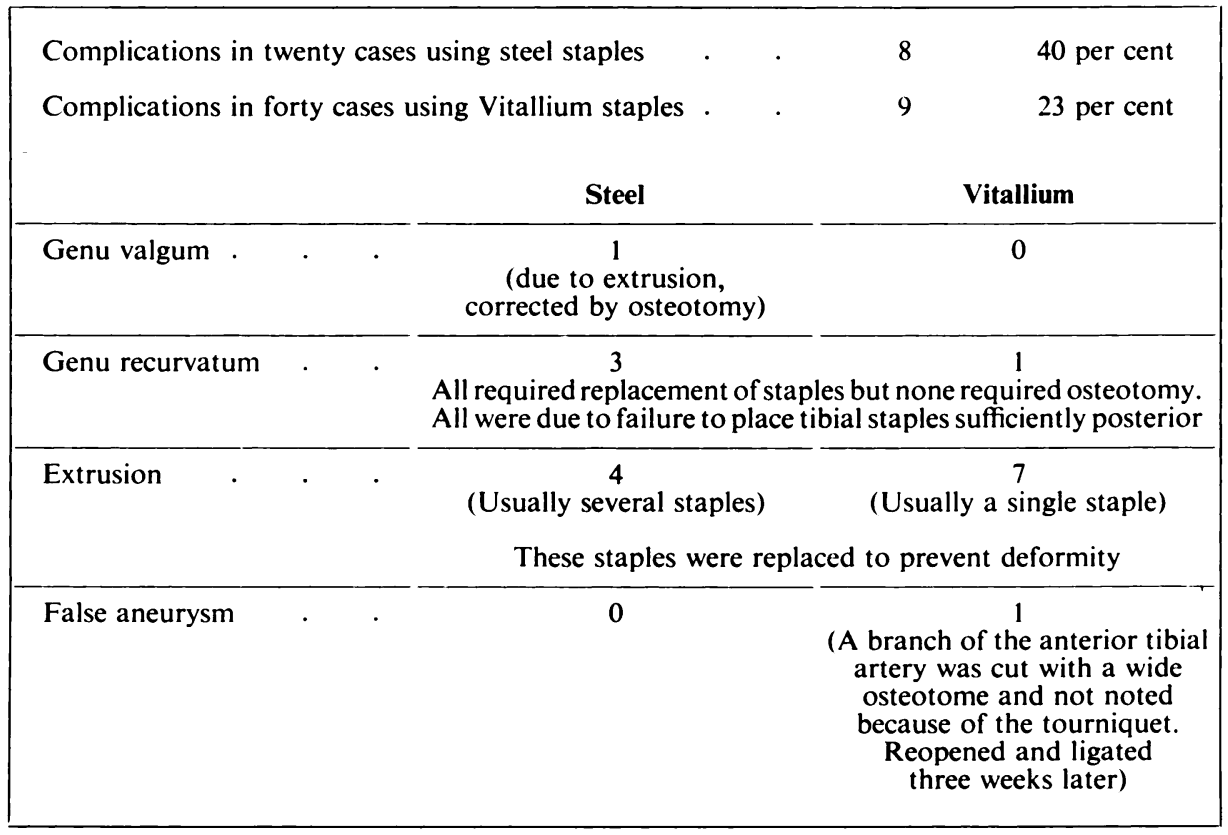

The absence of complications, of course, is only a prerequisite for an acceptable method of correcting leg length or angulation. That stapling will indeed cause growth retardation is a fact. Our studies do not refute anything stated by the advocates of stapling but do show that the surgeon not frequently confronted with length inequality may be unable to produce results to within acceptable limits using the published time tables. He must, therefore, make certain allowances both in timing and technique.

The question of premature fusion-The supreme advantage and sole indication for stapling over other forms of arrest is reversibility. In Blount's series $(1954,1957)$ of nearly 200 , premature fusion has never been a problem. Others report difficulties but that is perhaps because of differences in surgical technique or timing. For instance, an epiphysis stapled at the age of nine and released at the age of eleven may well have more potential to resume growth than one done at twelve and released at fourteen.

If, to remove a staple, it has to be dug out of a mass of bone, the remaining bony bridge is not dissimilar to the original Phemister epiphysiodesis (Phemister 1933; Brockway, Craig and Cockrell 1954), and one cannot say that the staples themselves are the cause of permanent arrest under these circumstances. Further, although the growth plate can withstand a great 
deal of injury, it may occasionally react to slight insult. The surgeon who ordinarily relies upon incision of the periosteum or probing with needles to locate the growth plate may, even then, misplace one or two staples; the damage is often more than is realised, with the result that the tendency to premature fusion is increased. Radiographic control and the use of spring calipers reduces this risk.

Incision of the periosteum to seat the staple is often recommended but encourages the development of a bone bridge. Proper angulation of staples against the intact periosteum gives sufficient anchorage of a Vitallium staple and none of our staples has become incorporated in bone, in contrast to the 25 per cent reported by Green and Anderson (1957). Lastly, removal of the staples without further injury is imperative, because periosteum and bone cannot be cut and crushed without producing callus.

The arbitrary two-year limit for retention of staples has been dropped. Goff (1960), however, has found closure of the epiphysial line following thirty-four to forty-four months of arrest by staples. In reviewing our radiographs we found a significant number of children whose epiphyses fused in both extremities somewhat before the expected chronological age of the bone, perhaps as a result of the underlying pathology. Thus a girl stapled at the age of ten might well show epiphysial fusion in a biopsy specimen taken forty-four months later, when she would be nearly fourteen, without blame being attached to the staples.

As a further safeguard for the doubtful, a child stapled at the age of ten or eleven should easily make up an inch to an inch and a half (Ratliff 1959) before thirty-four months have passed, if both the tibia and femur are stapled.

\section{SUMMARY}

1. The authors have found epiphysial stapling a satisfactory and helpful procedure for correction of limb length discrepancy and recommend its use as described.

2. The use of a spring caliper for absolute radiographic location of the epiphysial plates and the use of two Vitallium staples each side is recommended.

3. Suggestions for avoiding injury to the epiphyses are outlined.

4. The experience of stapling operations in seventy children is reported. Forty-four of these patients have completed growth.

\section{REFERENCES}

Blount, W. P. (1954): Discussion. Journal of Bone and Joint Surgery, 36-A, 1,069.

Blount, W. P. (1957): Discussion. Journal of Bone and Joint Surgery, 39-A, 872.

Blount, W. P. (1958): Unequal Leg Length in Children. Surgical Clinics of North America, 38, 1,107.

Blount, W. P., and Clarke, G. R. (1949): Control of Bone Growth by Epiphyseal Stapling. Journal of Bone and Joint Surgery, 31-A, 464.

Blount, W. P., and Zeier, F. (1952): Control of Bone Length. Journal of the American Medical Association, 148, 451.

Brockway, A., Craig, W. A., and Cockrell, B. R., Jun. (1954): End-Result Study of Sixty-two Stapling Operations. Journal of Bone and Joint Surgery, 36-A, 1,063.

CAssidy, R. H. (1958): Epiphyseal Injuries of the Lower Extremities. Surgical Clinics of North America, 38, 1,125.

Dalton, J. B., Jun., and Carpenter, E. B. (1954): Clinical Experiences with Epiphysial Stapling. Southern Medical Journal, 47, 544.

Goff, C. W. (1960): Histologic Evidence of Controls of Growth in the Femur and Tibia by Staples. Journal of Bone and Joint Surgery, 42-A, 186.

Green, W. T. (1949): Discussion. Journal of Bone and Joint Surgery, 31-A, 484.

Green, W. T., and Anderson, M. (1957): Epiphyseal Arrest for the Correction of Discrepancies in Length of the Lower Extremities. Journal of Bone and Joint Surgery, 39-A, 853.

Phemister, D. B. (1933): Operative Arrestment of Longitudinal Growth of Bones in the Treatment of Deformities. Journal of Bone and Joint Surgery, 15, 1.

Phemister, D. B. (1945): Discussion. Journal of Bone and Joint Surgery, 27, 34.

Ratliff, A. H. C. (1959): The Short Leg in Poliomyelitis. Journal of Bone and Joint Surgery, 41-B, 56.

Straub, L. R., Thompson, T. C., and Wilson, P. D. (1945): The Results of Epiphyseodesis and Femoral Shortening in Relation to Equalization of Limb Length. Journal of Bone and Joint Surgery, $27,254$.

White, J. W. (1949): Discussion. Journal of Bone and Joint Surgery, 31-A, 486. 